\title{
Herausforderungen aktueller IT-Entwicklungen
} Auswirkungen auf die Automatisierungstechnik

Virtualisierung, Cloud Computing und servicebasierte Architekturen sind Innovationen aus der IT, die langsam aber sicher in der Automatisierungstechnik Einzug halten. Auf der Namur-Hauptsitzung 2015 hat der Namur-Arbeitskreis 2.11 Industrielle IT/Leittechnik in einem Workshopbeitrag das Thema Automatisierungsysteme im Kontext aktueller IT-Entwicklungen aufgegriffen. Die Anforderungen der Automatisierungstechnik bezüglich Lebenszykluskonzepten und Verfügbarkeit bleiben bestehen und müssen mit den neuen Trends in Einklang gebracht werden. In diesem Beitrag werden die wichtigsten Aspekte dieser Entwicklungen dargestellt. Deren Nutzen und Risiken sollen 2016 mit den Anbietern diskutiert werden, um daraus Anwendungsempfehlungen und Unterstützungsmöglichkeiten für die Anwender abzuleiten.

\section{SCHLAGWÖRTER Automatisierungssysteme / Lebenszykluskonzepte / Obsoleszenzmanagement / Cloud Computing}

\section{Challenges of latest IT trends - Consequences for automation systems}

IT innovations such as virtualisation, cloud computing and service based architectures are also contributing to automation technology. At the Namur General Meeting 2015, the Namur Working Group 2.11 Industrial IT/Process Control Technology presented a workshop contribution on Automation systems in the context of current IT development. The requirements of automation technology concerning life cycle concepts and reliability remain valid and need to be aligned with those trends. Here, the most significant aspects of those developments are outlined. Their advantages and risks will be discussed with the suppliers in 2016. The objective will be to provide users with recommendations and support.

KEYWORDS automation systems / life cycle concepts / obsolescence management / cloud computing 


\section{THOMAS HAUFF, STEFAN SÖFFING, BASF}

W aren Automatisierungssysteme vor Jahren noch eigenständige proprietäre Einheiten, so haben sie sich heute zu komplexen, integrierten IT-Anwendungen entwickelt, die die (noch) proprietären Komponenten, wie Controller und I/O-Systeme, zunehmend über Standardschnittstellen einbinden. Auch für früher automatisierungsspezifische Themen, wie Integrität und hohe Verfügbarkeit der Komponenten, stehen heute Lösungen aus der IT bereit. Die Entwicklung hin zu einer derart engen Verzahnung mit der IT-Technologie trifft die Automatisierungstechnik mit allen damit verbundenen Vor- und Nachteilen.

\section{SYSTEME IM KONTEXT AKTUELLER IT-ENTWICKLUNGEN}

Die Dynamik der IT-Entwicklung ist nach wie vor hoch, und die Automatisierungtechnik profitiert gewiss von zahlreichen neuen Wegen, die in der IT eingeschlagen werden. Klar ist aber ebenso, dass die Anforderungen in der Automatisierung eine konservative Betrachtung der neuen Technologien erforderlich machen. Allerdings: Die Themen haben sich verschoben. Während früher Hardwarefragen und Betriebssystemplattformen intensiv diskutiert wurden, stellt sich heute die Frage nach Konzepten und Architekturen von IT-Systemen und wie diese sinnvollerweise für automatisierungstechnische Systeme verwendet werden können. Proprietär ist nur noch, was nicht geeignet mit Standard-IT umgesetzt werden kann.

Auch die Interessenslage der Anwender hat sich weiterentwickelt: Während Lebenszykluskonzepte und Stabilitätsfragen unverändert relevant sind, rücken weitere Themen in den Fokus: Servicekonzepte, Integrationsfragen und nachhaltiges Engineering werden zunehmend thematisiert. Vertikale Integration und Sicherheit der Automatisierungstechnik sind Themen, die immer mehr Einfluss auf das Kerngeschäft der Automatisierungstechnik nehmen. Dabei sind die Themen komplexer geworden und setzen mehr IT-Wissen voraus.
Das ist Grund genug, die Herausforderungen und Auswirkungen aktueller IT-Entwicklungen auf die Automatisierungstechnik zu beleuchten. Chancen sollen wahrgenommen werden, Risiken vermieden und den Anwendern eine Unterstützung angeboten werden.

\subsection{Vorgehen und Ziele der Aktivitäten}

In der Namur-Empfehlung NE 121 [1, 2] werden Qualitäts- und Lebenszyklusaspekte der Leittechnik behandelt. Vor dem Hintergrund der fortschreitenden ITEntwicklung ist es sinnvoll, die in der Empfehlung genannten Maßstäbe erneut anzuwenden. Diese Fragestellung spiegelt sich in den Aktivitäten des NamurArbeitskreis 2.11 Industrielle IT/Leittechnik, der sich folgenden Aspekten widmet:

- Sicherstellen der Wirtschaftlichkeit der Automatisierung,

- Nutzung von Chancen neuer Technologien,

- Hinweise auf mögliche Risiken neuer Technologien,

- Unterstützung der Anwender.

Das konkrete Ziel der aktuellen Tätigkeiten ist ein Arbeitsblatt zur Unterstützung der Anforderungs- und Lösungsspezifikation zwischen Herstellern/Lieferanten und Anwendern. Dabei sollen Lösungsansätze für die Problematik der Unterschiede zwischen typischen Automatisierungssystemzyklen und typischen Prozessanlagenzyklen, siehe Bild 1, aufgezeigt werden. Die aus der NE 121 ableitbaren Anforderungen sollen mit Blick auf IT-Aspekte aufgelistet und mit den Herstellern und Lieferanten diskutiert werden, indem aktuelle ITTrends an eben diesen Forderungen gespiegelt werden. Die Schwerpunkte dieser Betrachtungen werden sein:

- Möglichkeiten und Grenzen aktueller Lebenszykluskonzepte sowie zugehörige Normungsaktivitäten, Aspekte der Bewertung und Flexibilisierung bestehender Konzepte, 
- Virtualisierung als ein Mittel zur Entkopplung der Hardware-, Betriebssystem- und Systemsoftwarelebenszyklen,

- Nutzen und Risiken von servicebasierter Lizenzierung und Cloud-Konzepten bei Systemanwendungen und Betriebssystemen.

\section{SYSTEMUNTERSTÜTZUNG UND LEBENSZYKLUSMODELLE}

Es gibt sie noch: die proprietären Prozessleitsysteme mit ewigem Restsupport. Der Retro-Look alter Klötzchengrafik auf modernen hochauflösenden Bildschirmen mag mancher Prozessführungsstrategie gewisser Anlagentypen nicht im Wege stehen. Wenn die damals mit hohem Aufwand erzeugten Anwendungen ausreichen, Ersatzteile und Know-how noch verfügbar sind, sowie angemessene Automation-Security-Lösungen gefunden werden, dann steht der weiteren Nutzung dieser Systeme nichts entgegen.

Trotzdem ist die Nutzung von Standardsystemen nicht aufzuhalten, zumal der Ansatz viele Vorteile haben kann, zum Beispiel gerade bezüglich der genannten Automation-Security-Themen. Allerdings können Automatisierungssysteme auf Basis von Standard-IT schwerlich mit zu propietären Lösungen vergleichbaren Supportmaßstäben bemessen werden. Sie waren immer gekennzeichnet durch eine enge Kopplung zwischen Hardware, Betriebssystemen und Systemversionen. Trotz der deutlich geringeren Kosten im Vergleich zu proprietären Systemen, wurde der resultierende Migrationsaufwand oft kritisch gesehen. Die IT hatte Antworten: Virtualisierung ermöglicht ein Aufbrechen dieser Kopplung. Solange es eine Plattform gibt, die eine Installation eines älteren Betriebssystems ermöglicht, kann eine Migration auf neue Systemversionen vermieden werden, wenn die vorhandene Version noch ausreichend unterstützt wird.

Analoge Überlegungen greifen jedoch genauso in den Bereichen, die bislang noch durch rein proprietäre Lösungen geprägt sind: So ist im Bereich der Controller teilweise eine zunehmende Innovationsrate $\mathrm{zu}$ beobachten. Der damit einhergehenden potenziellen Nutzensteigerung stehen die Befürchtungen sinkender Supportzeiten entgegen.

\subsection{Phasenmodell}

Alle namhaften Hersteller von Automatisierungslösungen beschreiben die Lebensphasen ihrer Produkte mit ihrer zugehörigen Unterstützung in einem Lebenszyklusmodell. Die Notwendigkeit solcher Beschreibungen ergibt sich auch aus [3]. Ohne auf spezifische Ausprägungen einzugehen, soll im Beitrag eine generische Darstellung solcher Phasen gegeben werden:

- Bevorzugte Produkte = aktuell, neueste Generation

- Klassische Produkte = noch voll erhältliche Produkte

- Unterstützte Produkte = nicht mehr oder nur noch für Ersatzzwecke und kleine Erweiterungen erhältliche Produkte

- Obsolete Produkte = keine Unterstützung mehr

Die typische Unterstützung der Versionen der Systemsoftware ist gegliedert nach deren Haupt- und SubVersionsnummern:

- Die aktuelle Version wird in allen Sub-Versionen unterstützt,

- die vorhergehende Version wird nur in letzter SubVersion unterstützt.

Eine solche Sicht lässt sich ebenso aus dem IEC-Standard 62890 Life Cycle Management [3] ableiten. Gemäß dieses Standards sind solche Phasen sinnvoll und in vielen Fällen ausreichend. Allerdings geht dieser Standard davon aus, dass Anwender gemeinsam mit Herstellern und Lieferanten die Unterstützung in Einzelverträgen geeignet erweitern. Der Namur-AK 2.11 will ausloten, in welchen Grenzen das möglich, sinnvoll und praxisgerecht ist.

\subsection{Kritische Betrachtung des Phasenmodells}

Die anerkannte Gliederung des Phasenmodells, wie zuvor aufgeführt, bietet eine einfache und praktikable Methodik zur Darstellung der Lebenszyklen der Produkte. Der Investitionsschutz benötigt aber darüber hinausgehende, konkret zeitbezogene Zusagen über die Unterstützung von Systemen. Aussagen über Produktlebensyzyklen und Versionsunterstützung enthalten notwendige Definitionen, die um zeitliche Angaben ergänzt werden sollten. Das können Mindestverweildauern von Produkten in ihren Lebenszyklen sein (Beispiel: die Phase unterstützte Produkte umfasst mindestens zehn Jahre) oder Festlegungen über Versionsfolgen (Beispiel: neue Versionen erscheinen frühestens nach fünf Jahren).

Ein Beispiel mag verdeutlichen, dass der Zeitpunkt einer Beschaffung in Relation zur Lebenszyklusphase eines Produkts einen signifikanten Unterschied der Unterstützungszeit bewirken kann: Bei Beschaffung einer Automatisierungslösung, deren bevorzugte Produkte kurz vor Transfer nach klassisch stehen, kann die Zeit, während der das Produkt noch ausreichend verfügbar ist (beispielsweise für eine große Erweite- 
Verfahrenstechnische Anlage

Prozessleitsystem
3-4 Jahre IT-Hardware-Komponenten
Ca. 2 Jahre System-Software

BILD 1: Vergleich der typischen Nutzungszeit einer verfahrenstechnischen Anlage im Vergleich zu Prozessleitsystemen, Systemsoftware und IT-Hardware-Komponenten, siehe [1]
30 Jahre

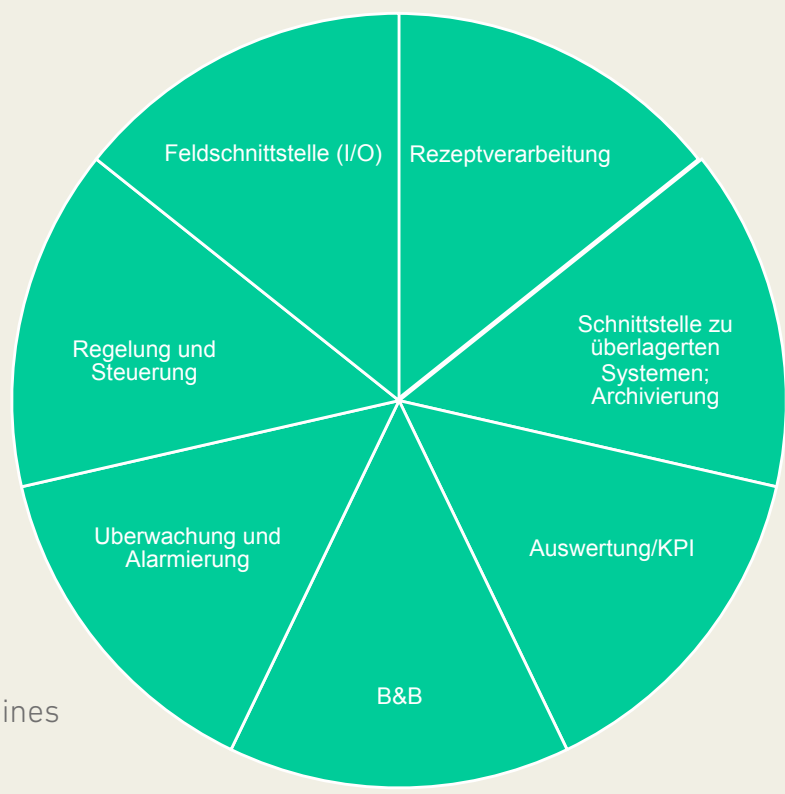

BILD 2: Funktionalitäten eines PLS in Anlehnung an [7]
Zu bedenken ist, inwieweit solche Zusagen ausreichend rückwärts integriert sind. Ein Beispiel ist die Frage nach der Lieferbarkeit weiterer Lizenzen für Erweiterungen in älteren Versionen: Lizenzen sind beim Originalhersteller oft nur für aktuelle Versionen erhältlich. mitiert. Der Einsatz der neuen bevorzugten Produkte erfordert aber möglicherweise eine Migration des bereits vorhandenen Leitsystems.

Relativ dazu ist die Unterstützung von Systemsoftware mit Blick auf die zuvor beschriebene Versionsfolge sogar eher noch kritischer zu sehen: Wird eine Automatisierungslösung in einer bestimmten Version beschafft, so ist in Anbetracht der Projektlaufzeit oft schon nach einer Betriebszeit von wenigen Jahren eine neue Version verfügbar. Ist die installierte Version nicht auf Letztstand, kann bereits dann eine Hochrüstung erforderlich sein. Dabei ist die ausschließliche Unterstützung des Letztstandes einer Vorversion oft eine unnötige Härte, denn sie erzwingt meist vermeidbare Hochrüstungen. Solche, typischerweise zirka fünf Jahre alten, Installationen sind bewährt. Solange sie nicht wesentlich erweitert werden, kann der Anwender akzeptieren, dass die Entwicklungsunterstützung von älteren Subständen der Vorversionen reduziert wird. Eine Unterstützung bei typischen Serviceinsätzen sollte aber dennoch möglich sein. Einzelabsprachen zwischen Anwendern und Herstellern beziehungsweise Lieferanten ermöglichen in solchen Fällen eine Ausdehnung der Unterstützung.

\subsection{Flexibilisierung der Unterstützung}

Neue Anforderungen (zum Beispiel regulative Vorgaben) an den Anwender einer Automatisierungslösung erfordern häufig neue Funktionsmodule (beispielsweise als Teil des Audit Trails). Wenn diese nicht im Sinne eines modularen Aufbaus auf Basis der installierten Version implementiert werden können, entsteht der Bedarf nach Migrationen. Bei zertifizierungspflichtigen Produktionen übersteigen die Zertifizierungskosten einer solchen Migration sehr schnell die Implementierungskosten um ein Mehrfaches. Funktionale Unterstützung und Ertüchtigung auch älterer Stände ist daher aus Sicht der Anwender wünschenswert.

Die Betrachtung der Kosten aus Sicht des Anwenders erfordert indes, aber ebenso aus anderen Gründen, tiefergehende Überlegungen: So sind neben den reinen Hochrüstungskosten auch die Stillstandskosten zu sehen. Für den Anwender ist eine Flexibilität der Gestaltung der Migrationsphasen sehr wichtig, damit solche Maßnahmen, zum Beispiel mit Um- 
bauten und ohnehin notwendigen Stillständen, verbunden werden können.

Wesentliche Verlängerungen der Lebenszyklen sind auf Grund der Dynamik der IT-Entwicklung sicherlich kaum zu erwarten. Eine Optimierung der Migrationskosten lässt sich aber durch aufwandsarme Teilmodernisierungen erreichen, insbesondere, wenn diese im laufenden Betrieb online durchgeführt werden können.

Das Phasenmodell ist damit ein wichtiges Element eines Obsoleszenzmanagements des Herstellers, aber nicht das Obsoleszenzmanagement selbst. Der NamurAK 2.11 wird sich nun unter anderem mit folgenden Fragen beschäftigen:

- Ist es innerhalb der Produkte eines Herstellers möglich, Teilfunktionalitäten aufwandsarm zu ersetzen (zum Beispiel Controller)?

- Ist eine solche Austauschmöglichkeit Teil des Lebenszykluskonzepts des Herstellers und damit ein Designziel des Systems?

- Welchen Nutzen hätten neue modulare Einbindungstechnologien für den Lebenszyklussupport (siehe [4-6])? Ein mittels Module Type Package (MTP) eingebundenes I/O-System wäre leichter ersetzbar und könnte leichter als Subystem eines Nachfolgesystems dienen.

Diese Fragen sollten eigentlich nicht neu sein: Die Tauchnitz-Torte, siehe Bild 2, hat schon vor 20 Jahren einen modularen Ansatz postuliert [7], wenn auch mit etwas anderer Absicht, denn damals ging es eher um die Frage der Zusammenschaltung von Modulen verschiedener Hersteller.

\subsection{Bewertungsverfahren von Lebenszykluskonzepten}

Während des Lebenszyklus des PLS ist ein partieller Austausch von Leitsystemkomponenten unvermeidbar. Damit verbundene Kosten gilt es möglichst bereits zum Zeitpunkt der Investition abzuschätzen und als Teil der Investitionsentscheidungen zu bewerten. Ein gängiges Werkzeug der betriebswirtschaftlichen Investitionsrechnung ist die Kapitalwertmethode (net present value, NPV) [8].

Obwohl die Kapitalwertmethode eine anerkannte Möglichkeit zur Investitionsbewertung darstellt, sei angemerkt, dass die Wahl des zu Grunde gelegten Zinssatzes (der Kalkulationszinsfuß) mit großer Unsicherheit belastet sein kann. Gleichzeitig hat der Zinsfuß jedoch einen erheblichen Einfluss auf das Ergebnis der Bewertung und muss deshalb mit größter Sorgfalt gewählt werden. Dazu gehört insbesondere eine Betrachtung der individuellen Risiken im Rahmen der Investition. In jedem Fall aber ist zu beachten, dass die idealisierte Kapitalwertmethode auf der fiktiven Annahme eines vollkommenen Kapitalmarktes beruht [9], das heißt Soll- und Habenzinsen müssten gleich sein - die real existierende erhebliche Spanne zwischen Soll- und Habenzins am Markt ist bei der Wahl des Kalkulationszinsfußes zu berücksichtigen.

Gerade vor dem Hintergrund dieser Unwägbarkeiten ist die Aussagekraft einer rein monetären Einschätzung mit Vorsicht zu betrachten: Auch wenn die Reinvestitionskosten für Teilersatz nach 15 Jahren möglicherweise nur geringen Einfluss auf den NPV der Leitsystemkosten zum Investitionszeitpunkt haben, so erscheint die Situation nach 15 Jahren, wenn die Reinvestition fällig wird, in einem ganz anderen Licht. Einer strategischen Bewertung der Kosten für den Erhalt der Betriebsfähigkeit muss somit eine wesentliche Rolle bei der Kostenbewertung zum Investitionszeitpunkt zukommen.

Zur Unterstützung der Anwender bietet die NE 121 Bewertungskriterien für Automatisierungslösungen, diese sollten jedoch für den aktuellen Stand der ITEntwicklung konkretisiert werden.

\section{3. ÜBERTRAGUNG VON IT-KONZEPTEN}

Ohne Zweifel bringt die Entwicklung in der IT viele interessante Ideen mit sich, die im Kontext der Automatisierungstechnik vorteilhafte Anwendung finden könnten, sofern sie mit den speziellen Anforderungen der Prozessführung vereinbar sind. In jedem Fall aber hat die sich ständig und immer schneller verändernde IT Auswirkungen auf althergebrachte Denkweisen in der Leitsystemtechnik. Diese zu beleuchten, soll Gegenstand der Arbeit des Namur-AK 2.11 in 2016 sein.

\subsection{Virtualisierung}

Virtualisierung entkoppelt die Computerhardware von der Softwareschicht - bestehend aus (Nutz-)Betriebssystem und Anwendungen - durch Einführung einer virtuellen Hardware, siehe Bild 3. Der Einsatz virtueller Maschinen (VM) an Stelle dedizierter klassischer Hardware kann dabei verschiedene Zwecke verfolgen:

- Bessere Ausnutzung der Hardware,

- Erhöhung der Verfügbarkeit durch hochwertigere Hardwareplattformen,

- Erhöhung der Verfügbarkeit durch Nutzung der Redundanz- und Reparaturmechanismen der Virtualisierungsplattformen,

- Effizienzsteigerung des Service: Kopieren von Images statt Installation von Systemen,

- Längerer Betrieb von Systemen nach Abkündigung dedizierter Hardware. 


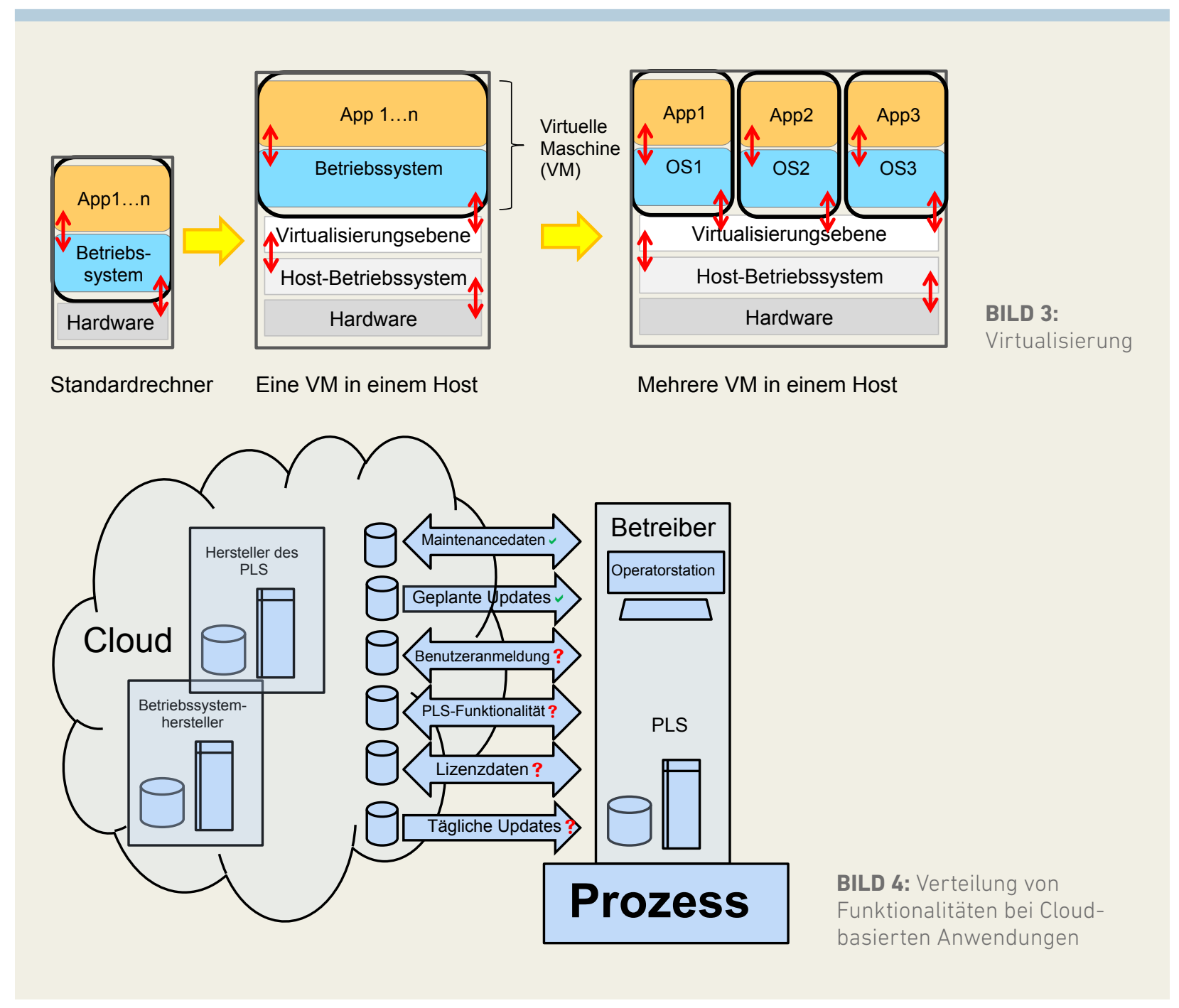

Der Nutzen dieser Technik ist heute anerkannt. Jedoch gibt es einige Aspekte, die eine nähere Diskussion mit den Herstellern und Lieferanten von Automatisierungstechnik erfordern und die der NamurAK 2.11 daher bei seinen geplanten Aktivitäten berücksichtigen wird:

- Wie erfolgt die Einbindung der Überwachungsmechanismen der Virtualisierungsplattform in die Systemdiagnose der Automatisierungssoftware? Wird der Anwender der Automatisierungslösungen mit den Details dieser Technologie zusätzlich belastet oder wird das Virtualisierungssystem in die Systemdiagnose einheitlich eingebettet?

- Wie sind die Geschäftsmodelle des Lieferanten der Virtualisierungslösung: Ist die Unterstützung äl- terer Betriebssystemvarianten noch im Fokus? Kann die zuvor erwähnte Unterstützung älterer Betriebssysteme nach Abkündigung dedizierter Hardware auf diese Weise erfolgen oder ist zu erwarten, dass dieser Aspekt der Virtualisierung aus dem Fokus gerät?

\subsection{Servicebasierte Automatisierungslösungen}

Die rasante Entwicklung in der Informationstechnologie, verbunden mit dem stetig wachsenden Anspruch an die Innovationsrate im Softwarebereich, führte in den letzten Jahren zu immer kürzeren Releasezyklen von Updates bezüglich Funktionalität und Behebung von Sicherheitslücken. Nicht zuletzt 
trägt dies der massiv steigenden Bedrohung jedweder Informationssysteme durch Cyberangriffe Rechnung. Die im Softwaremarkt für Heim- und Büroanwendungen etablierten Mechanismen wirken sich auf alle Bereiche der Softwareentwicklung aus: Getragen von den technischen Möglichkeiten, große Softwareupdates über das Internet zu verbreiten, gilt es, Software zu jedem Zeitpunkt mit allen jeweils neuesten Funktionalitäten zur Verfügung zu stellen.

Bis vor wenigen Jahren wurde Software üblicherweise als abgeschlossenes Paket mit definiertem Funktionsumfang und klassischer Versionsführung ausgeliefert - zwischen großen Versionssprüngen mit Änderungen am Funktionsumfang werden vorzugsweise kleinere Fehlerbehebungen veröffentlicht. Die-

\section{REFERENZEN}

[1] Hauff, T.: Qualitätssicherung leittechnischer Systeme. atp - Automatisierungstechnische Praxis 51(1-2), S. 72-29, 2009

[2] NE 121: Qualitätssicherung leittechnischer Systeme. Namur, 2008

[3] IEC-62890: Life Cycle Management, in Vorbereitung, TC 65, IEC 2015

[4] Urbas, L.; Bleuel, S.; Jäger, T.; Schmitz, S.; Evertz, L.; Nekolla, T: Automatisierung von Prozessmodulen. atp edition - Automatisierungstechnische Praxis 54(1-2), S. 44-53, 2012

[5] Obst, M.; Holm, Th.; Bleuel, St.; Claussnitzer, U.; Evertz, L.; Jäger, T.; Nekolla, T.; Pech, St.; Schmitz, St.; Urbas, L.: Automatisierung im Life Cycle modularer Anlagen. Veränderungen und Chancen. atp edition - Automatisierungstechnische Praxis 55(1-2), S. 24-31, 2013

[6] Obst, M.; Holm, T.; Urbas, L.;Fay, A.; Kreft, S.; Hempen, U.; Albers, T.: Beschreibung von Prozessmodulen. atp edition - Automatisierungstechnische Praxis 57(1-2), S. 58-59, 2015

[7] Tauchnitz, Th.: Die „neuen Prozeßleitsysteme“ - wohin geht die Reise? atp - Automatisierungstechnische Praxis 38(11), S. 12-23, 1996

[8] Kruschwitz, L., Husmann, S.: Finanzierung und Investition. Oldenbourg Wissenschaftsverlag 2009

[9] Keitel, D.: Die Kapitalwertmethode und die Grenzen Ihrer Anwendung. GRIN Verlag 2007

[10] Benlian, A.; Hess, T.; Buxmann, P.: Software-as-aService. Springer Fachmedien 2010

[11] Upgrade für Windows 10. c't 27 S. 70-78, Heise, 2015 [12] Arch Linux leicht gemacht. c't 27 S. 164-167, Heise, 2015 se Denkweise wird mehr und mehr verdrängt durch einen stetigen Weiterentwicklungsprozess. Getrieben durch die Entwicklungen im Cloud Computing, siehe Bild 4, bei dem Funktionen und Daten nicht mehr lokal, sondern an zentralen Stellen vorgehalten werden, gipfelt dies schließlich im Software-as-a-Service (SaaS), siehe zum Beispiel [10]: Softwareanwendungen werden damit nur noch als Dienstleistung zur Verfügung gestellt, die kontinuierliche Wartung, Erweiterung und Fehlerbehebung erfolgt transparent durch den Serviceanbieter. Die Anwendung ist gar nicht mehr lokal ablauffähig, sondern sie benötigt einen permanenten Zugang zu Cloud-Ressourcen. Es stellt sich die Frage, welche Funktionen sinnvollerweise in die Cloud verlagert werden können, ohne die Verfügbarkeit zu beeinträchtigen. Im Extremfall könnten sogar die Benutzerdaten oder Teile der PLSFunktionalität in der Cloud liegen, siehe Bild 4.

Inzwischen weitet sich dieser Trend bis hin zur Ebene des Betriebssystems aus. Auch wenn die Entwicklung noch am Anfang steht, ist absehbar, dass traditionelle Kompatibilitätsaussagen (Version y der Anwendung x wurde getestet auf dem Betriebssystem Version z) durch das dynamische Versionierungssystem im Kontext von SaaS an ihre Grenzen gebracht werden. Gegebenenfalls haben Betriebssysteme gar keinen definierten Versionsstand sondern sind tagesaktuell [11, 12]. Daraus ergibt sich eine nicht $\mathrm{zu}$ vernachlässigende Herausforderung für hoch verfügbare Systeme, wie sie typischerweise im Automatisierungsumfeld eingesetzt werden: Bereits die Installation eines Systems erfordert hundertprozentige Reproduzierbarkeit des Softwarestands, das heißt bekannterweise verlässliche und getestete Softwarekombinationen. Während des Lebenszyklus stellt die kontinuierliche Hochrüstung ferner eine potenzielle Gefährdung für den stabilen Betrieb der Systeme dar.

Ausgehend von diesen Herausforderungen hat sich die Erkenntnis durchgesetzt, dass bei permanenter Weiterentwicklung eine Langzeitunterstützung reproduzierbarer Versionsstände unumgänglich ist. Diese werden typischerweise im Rhythmus von drei Jahren veröffentlicht, bieten zehn Jahre Support und erfahren während der Laufzeit ausschließlich sicherheitskritische Updates. Die Praktikabilität dieser Vorgehensweise muss sich allerdings erst noch bewähren, insbesondere da die für den Anwender tatsächlich nutzbare Lebensdauer mit großer Wahrscheinlichkeit nicht wesentlich über fünf bis sieben Jahre hinausgehen wird - vor allem, wenn die übliche Einführungszeit am Markt im Rahmen der Versionspolitik berücksichtigt wird. Hier gilt es abzuwägen, ob und wie der As-a-Service-Ansatz bei Betriebssystemen für die Automatisierungstechnik seine Vorteile ausspielen kann. 
Vor diesem Hintergrund will der Namur-AK 2.11 Rückmeldungen einholen, wie die Hersteller mit diesen Themen umgehen. Servicebasierte Softwareangebote kommen dabei an mehreren Stellen vor: Einerseits können die Automatisierungssysteme selbst auf Softwarepaketen (zum Beispiel Betriebssystemen, Office-Anwendungen und anderen Plattformen) basieren, die als Service lizenziert sind. Das kann zu Abhängigkeiten führen, denn ein Betrieb über die vom Hersteller dieser Pakete vorgegebenen Termine hinaus kann dann technisch oder rechtlich unmöglich sein, das heißt es entsteht möglicherweise die Notwendigkeit zur Migration. Ferner könnte eine temporäre Unverfügbarkeit von Lizenzierungsservern oder Cloud-Funktionen der Hersteller dieser Pakete zu Ausfällen der Automatisierungsfunktionen führen. Andererseits könnte der Hersteller der Automatisierungssysteme selbst solche Lizenzierungsmodelle wählen. Die Auswirkungen solcher Ansätze sollen geklärt und dargestellt werden.

\section{METHODIK}

Die geplante Vorgehensweise des Namur-AK 2.11 zur Bearbeitung dieser Themen soll sich an der NE 121 orientieren. Es ist vorgesehen, einen Fragenkatalog mit folgenden Schwerpunkten auszuarbeiten: Welche Ansätze haben die Hersteller und Lieferanten zur Kosten-/Aufwandsminimierung in Anbetracht der Konsequenzen der schnellen IT-Entwicklungen? Welche Vorteile können die Anwender daraus ziehen, was müssen sie berücksichtigen? Beispiele solcher Fragen sind:

- Gibt es minimale Zeiten zwischen Versionssprüngen?

- Gibt es minimale Zeiten der Produktunterstützungsphasen?

- Welche vertraglichen Regelungen zur anwenderspezifischen Längerunterstützung von Systemen außerhalb des Phasenmodells sind vorgesehen?

- Helfen modulare Einbindungstechnologien (MTP) zur Optimierung der Lebenszyklusunterstützung?

- Was sind die langfristigen Erwartungen an Virtualisierung?

- Welche Möglichkeiten von Cloud-Anwendungen sind im Kontext der Automatisierungstechnik nutzbringend anwendbar?

- Konkrete Frage: „Wir wollen n Jahre ununterbrochenen Betrieb einer Anlage gewährleisten was müssen wir beachten?“

\section{FAZIT}

Der Namur-AK 2.11 will als Schwerpunkt für 2016 den aktuellen Stand der IT-Entwicklung an der NE 121 spiegeln und mit den Herstellern und Anwendern solcher Systeme diskutieren. Schwerpunkte sind:

- Lebenszykluskonzepte unter besonderer Berücksichtung von Modularisierung und Teilmodernisierung,

- Virtualisierungskonzepte,

- Servicebasierte Lizenzmodelle und Cloud-Technologien,

- Strategische Bewertung von Systemkonzepten.

Chancen sollen wahrgenommen werden, Risiken vermieden werden und den Anwendern eine Unterstützung angeboten werden.

MANUSKRIPTEINGANG 17.12.2015

Im Peer-Review-Verfahren begutachtet

\section{AUTOREN}

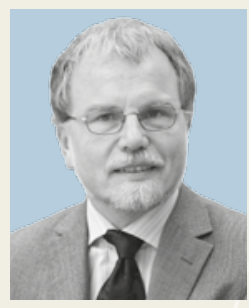

Dr. THOMAS HAUFF

(geb. 1960) ist Fachgruppenleiter im Fachzentrum Automatisierungstechnik in der BASF SE, Ludwigshafen. Seine Arbeitsschwerpunkte sind Qualitätssicherung in der Prozessleittechnik und Prozessleitsystemen. In der Namur leitet er den AK 2.11 „Industrielle IT/Leittechnik“.

BASF AG, GTG/ED - L440,

D-67056 Ludwigshafen,

Tel. +49 (0) 62160203 26,

E-Mail: thomas.hauff@basf.com

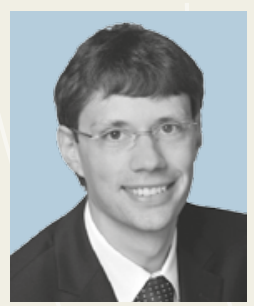

Dr. STEFAN SÖFFING (geb. 1984) ist seit 2012 bei der BASF SE, Ludwigshafen, im Fachzentrum Automatisierungstechnik tätig. Seine Arbeitsschwerpunkte sind Projekte im Bereich komplexer Schnittstellen und der Batchautomatisierung. 